\title{
In vitro derivation of functional insulin-producing cells from human embryonic stem cells
}

\author{
Wei Jiang ${ }^{1, *}$, Yan Shi ${ }^{1,5, *}$, Dongxin Zhao ${ }^{1}$, Song Chen ${ }^{1}$, Jun Yong ${ }^{1}$, Jing Zhang ${ }^{2}$, Tingting Qing ${ }^{1}$, Xiaoning Sun ${ }^{1,3}$, \\ Peng Zhang ${ }^{1}$, Mingxiao Ding ${ }^{1}$, Dongsheng Li $^{4}$, Hongkui Deng ${ }^{1,2,3}$ \\ ${ }^{1}$ Department of Cell Biology and Genetics, College of Life Sciences, Peking University, Beijing 100871, China; ${ }^{2}$ Beijing Laboratory \\ Animals Research Center, Beijing 100012, China, ${ }^{3}$ Laboratory of Chemical Genomics, Shenzhen Graduate School of Peking Univer- \\ sity, the University Town, Shenzhen 518055, China; ${ }^{4}$ Provincial Key Laboratory of Embryonic Stem Cell Research, Tai-He Hospital \\ Yunyang Medical College, 32 S. Renmin Rd., Shiyan 442000, China
}

The capacity for self-renewal and differentiation of human embryonic stem (ES) cells makes them a potential source for generation of pancreatic beta cells for treating type I diabetes mellitus. Here, we report a newly developed and effective method, carried out in a serum-free system, which induced human ES cells to differentiate into insulin-producing cells. Activin A was used in the initial stage to induce definitive endoderm differentiation from human ES cells, as detected by the expression of the definitive endoderm markers Sox17 and Brachyury. Further, all-trans retinoic acid (RA) was used to promote pancreatic differentiation, as indicated by the expression of the early pancreatic transcription factors $p d x l$ and $h l x b 9$. After maturation in DMEM/F12 serum-free medium with bFGF and nicotinamide, the differentiated cells expressed islet specific markers such as C-peptide, insulin, glucagon and glut2. The percentage of C-peptide-positive cells exceeded $15 \%$. The secretion of insulin and C-peptide by these cells corresponded to the variations in glucose levels. When transplanted into renal capsules of Streptozotocin (STZ)-treated nude mice, these differentiated human ES cells survived and maintained the expression of beta cell marker genes, including C-peptide, pdx1, glucokinase, nkx6.1, $I A P P$, pax 6 and $T c f 1$. Thirty percent of the transplanted nude mice exhibited apparent restoration of stable euglycemia; and the corrected phenotype was sustained for more than six weeks. Our new method provides a promising in vitro differentiation model for studying the mechanisms of human pancreas development and illustrates the potential of using human ES cells for the treatment of type I diabetes mellitus.

Keywords: human embryonic stem cell; direct differentiation; insulin-producing cell; diabetes

Cell Research (2007) 17:333-344. doi: 10.1038/cr.2007.28; published online 10 April 2007

\section{Introduction}

Human pancreatic islet transplantation at present is the preferred therapeutic option for type I diabetes [1].

\footnotetext{
* These two authors contributed equally to this work.

${ }^{5}$ Present address: The Scripps Research Institute, Chemistry Department, SP3130, 10550 North Torrey Pines Road, La Jolla, CA, USA.

Correspondences: Hongkui Deng ${ }^{1}$, Dongsheng $\mathrm{Li}^{2}$

${ }^{1}$ Tel: 86-10-6275-6954; Fax: 86-10-6275-6954

E-mail: hongkui_deng@pku.edu.cn

${ }^{2}$ Tel: 86-719-8801418; Fax: 86-719-8801418

E-mail: dsli@yymc.edu.cn

Received 12 March 2007; revised 13 March 2007; accepted 13 March 2007; published online 10 April 2007
}

However, this therapy is not widely utilized because of the severe shortage of donor islets [2]. Human embryonic stem (ES) cells can be maintained in vitro for extended periods without loss of genetic stability, and are potential sources for generating a variety of specialized human cells needed for clinical applications. They hold the promise of serving as a source of insulin-producing donor cells in type I diabetes cell therapy $[3,4]$.

It has been reported that human ES cells can spontaneously differentiate into insulin-producing cells in vitro $[5,6]$. A five-stage protocol similar to that described by Lumelsky et al. [7] has been used to induce human ES cell differentiation into insulin-producing cells [8]. However, further investigation is needed to confirm that human ES 
cells are indeed induced to become functional insulinproducing cells by these methods because of a possibility of exogenous insulin uptake [9] and the lack of functional demonstration by an in vivo transplantation assay. Recently, D'Amour et al. reported a stepwise protocol, by which they obtained pancreatic hormone-expressing endocrine cells, and the purified insulin-expressing cells had an insulin content approaching that of adult islets [10]. However, the cells induced by their protocol released only a minimal amount of C-peptide in response to glucose stimuli. Furthermore, in vivo transplantation studies were not performed. Therefore, more effective approaches are required for inducing functional insulin-producing cells from human ES cells, which ideally should rescue the diabetic phenotype in vivo in an animal model.

In our previous study, we developed a novel three-stage induction method to induce mouse ES cells into insulinproducing cells, which could rescue diabetic mice after transplantation [11]. Here, a modified approach in serumfree culture medium has been adopted to induce human ES cells to differentiate into functional insulin-producing cells by combining activin A, all-trans retinoic acid (RA) in chemically defined medium (CDM), and other maturation factors such as bFGF and nicotinamide in DMEM/F12. Using this method, which consisted of an incubation with activin A and RA in chemically defined medium (CDM) followed by maturation in DMEM/F12 serum-free medium supplemented with bFGF and nicotinamide, we showed that the human ES cell-derived cells expressed islet specific genes such as $p d x 1$, insulin, C-peptide, glut2, glucagon, and amylase. The C-peptide-positive cells were TUNEL-negative and the percentage of C-peptide-positive cells achieved was more than $15 \%$. The secretion of insulin by these cells was responsive to variations in glucose levels. After transplantation into diabetic nude mice, $30 \%$ of the animals showed an obvious rescue of their hyperglycemia phenotype, and this condition was maintained for more than six weeks.

\section{Materials and Methods}

\section{Human ES cell culture and differentiation}

The human ES cell lines H1 and H9 (from WiCell Research Institute, Inc.) were cultured in an undifferentiated state as described [3]. We carried out all experiments in parallel with both cell lines. For differentiation, our protocol was designed as follows: in the first step, human ES cells were passaged onto 1\% Matrigel (B\&D Biosciences)-coated tissue culture dishes (Nunc). Then, the culture medium was changed to modified CDM: 50\% IMDM (Gibco) plus 50\% F12 NUT-MIX (Gibco), supplemented with Insulin-TransferrinSelenium-A (1:100, Gibco) and $450 \mu \mathrm{M}$ monothioglycerol (Sigma), and $5 \mathrm{mg} / \mathrm{ml}$ albumin fraction V (Sigma) [12] or X-Vivo10 (Cambrex) supplemented with $55 \mu \mathrm{M}$ 2-Mercaptoethanol (Gibco) and $0.1 \%$ albumin fraction V (Sigma). Two days later, the cells were induced with CDM containing $50 \mathrm{ng} / \mathrm{ml}$ activin A (Sigma) for $4 \mathrm{~d}$. After $4 \mathrm{~d}$ of activin A induction, the cells were transferred into CDM with $10^{-6} \mathrm{M}$ RA (Sigma) for another $4 \mathrm{~d}$. Then, the culture medium was changed from CDM to modified islet maturation medium [13]: DMEM/F12 (Gibco), Insulin-Transferrin-Selenium-A (1:100, Gibco) and $2 \mathrm{mg} / \mathrm{ml}$ albumin fraction $\mathrm{V}$ (Sigma) with $10 \mathrm{ng} / \mathrm{ml} \mathrm{bFGF} \mathrm{(Invitrogen)} \mathrm{for} \mathrm{the}$ first $3 \mathrm{~d}$ and with $10 \mathrm{mM}$ nicotinamide (Sigma) for the next $5 \mathrm{~d}$. The cells were then digested by $0.5 \mathrm{mg} / \mathrm{ml}$ dispase (Gibco) and transferred into Ultra Low Attachment culture dishes (Costar) for $5 \mathrm{~d}$ to achieve islet maturation in suspension culture.

\section{RT-PCR and Real-Time PCR analysis}

Total RNA was extracted by TRIzol Reagent (Invitrogen) from the cells during the induction process and from kidneys of transplanted mice. RNA was then reverse-transcribed into cDNA by MMLV reverse transcriptase (Promega).

PCR was performed with Ex Taq polymerase (TaKaRa) following the manufacturer's protocol. The cycle conditions were as follows: $94{ }^{\circ} \mathrm{C}$ for $5 \mathrm{~min}$ followed by 35 cycles $\left(94{ }^{\circ} \mathrm{C}\right.$ denaturation for 50 $\mathrm{S}, 56-58^{\circ} \mathrm{C}$ annealing for $30 \mathrm{~S}, 72{ }^{\circ} \mathrm{C}$ elongation for $\left.40 \mathrm{~S}\right)$, with a final incubation at $72{ }^{\circ} \mathrm{C}$ for $4 \mathrm{~min}$. The primers are shown in the supplemental information, Table $\mathrm{S} 1$.

Real-time PCR analysis was performed on ABI PRISM 7300 Sequence Detection System using the SYBR Green PCR Master Mix (TOYOBO). The PCR reaction consisted of $12.5 \mu \mathrm{l}$ of SYBR Green PCR Master Mix, $1 \mu \mathrm{l}$ of $10 \mu \mathrm{M}$ forward and reverse primers, $10.5 \mu \mathrm{l}$ of water, and $1 \mu \mathrm{l}$ of template cDNA in a total volume of $25 \mu 1$. Cycling was performed using the default conditions of the ABI 7300 SDS Software 1.3.1: $95^{\circ} \mathrm{C} 2 \mathrm{~min}$, followed by 35 cycles of $95^{\circ} \mathrm{C}$ for $15 \mathrm{~s}$ and $60^{\circ} \mathrm{C}$ for $1 \mathrm{~min}$. The relative expression of each gene was normalized against gapd. The primers of assayed genes, including $\beta$-actin, glucokinase, nkx6.1, IAPP, pax6 and Tcf1, were designed specifically for human genes. The primer sequences are shown in the supplemental information, Table S2.

\section{Immunohistochemistry assay}

Induced cells were fixed in 4\% paraformaldehyde and washed three times by PBS, then incubated with PBS containing $0.3 \%$ TritonX-100 (Sigma) and 10\% normal serum for $40 \mathrm{~min}$ at room temperature. Especially for nuclear antigens, the concentration of TritonX-100 was adapted to $0.5 \%$ and incubation time to 1 hour. The cells were then incubated with the primary antibody: Goat anti-human Sox 17 antibody (1:100, R\&D SYSTEMS), Mouse anti-human insulin monoclonal antibody (1:100, CHEMICON), Rabbit anti-human C-peptide antibody (1:200, Linco), glucagon antibody (Rabbit polyclonal IgG, 1:200, Santa Cruz), pdx1 antibody (Rabbit or Goat polyclonal IgG, 1:200, from Dr. C Wright), amylase antibody (Rabbit polyclonal IgG, 1:500, Sigma), ngn3 antibody (Goat polyclonal IgG, 1:200, Santa Cruz) or somatostatin antibody (Rabbit polyclonal IgG, $1: 200$, Santa Cruz), overnight at $4{ }^{\circ} \mathrm{C}$, and further incubated with the respective secondary antibody: TRITC-conjugated goat anti-rabbit IgG, FITC-conjugated rabbit anti-goat IgG, FITC-conjugated Goat anti-Mouse IgG (1:200, Santa Cruz), FITC-conjugated Donkey anti-goat IgG or TRITC-conjugated Donkey anti-rabbit IgG (1:200, Jackson ImmunoResearch, INC.). In all immunochemistry assays negative staining controls were carried out by omitting the primary antibody. Nuclei were detected by DAPI (Sigma) staining. Images were captured using Olympus microscope IX-71, LEICA microscope DM4000B or LEICA confocal microscope TCS-SP2.

The percentage of Sox17-, pdx1- and C-peptide-positive cells were calculated using Image-Pro Plus software (Media Cybernetics) 
as follows: an area to be measured was outlined by a tracer, with the number of counting frames preset so that the scope sampled the measuring sites randomly. The software counted the value of the respective chosen area. We utilized DAPI staining to estimate the amount of total cells. For counting the percentage of pdx1- or C-peptide-positive cells, the positive areas were estimated by the software in the same way. The percentage was calculated by the following formula: C-peptide (or pdx1) positive area/whole DAPI positive area. Pictures were taken from at least three independent cultures and fifty random different scopes of the differentiated cells to estimate the positive cell percentage.

The DeadEnd ${ }^{\mathrm{TM}}$ Fluorimetric TUNEL System (Promega) was utilized to perform the cell apoptosis assay. Images were captured using LEICA confocal microscope TCS-SP2.

\section{ELISA assay}

To test whether the insulin release of differentiated cells was glucose-dependent, two glucose concentrations $(2.5 \mathrm{mM}$ and 27.5 $\mathrm{mM}$ ) were used. After pre-incubation with Krebs-Ringer buffer at $37^{\circ} \mathrm{C}$ for $90 \mathrm{~min}$, the cells were incubated with Krebs-Ringer buffer containing $2.5 \mathrm{mM}$ glucose at $37^{\circ} \mathrm{C}$ for $15 \mathrm{~min}$. To induce insulin release, the cells were incubated with $27.5 \mathrm{mM}$ glucose for another 15 $\mathrm{min}$. Then the respective conditioned media were collected and tested for the content of released insulin with a Rat/Mouse Insulin ELISA Kit (Linco). To determine the C-peptide content in the differentiated cells, we treated the cells with lysis buffer: $50 \mathrm{mM}$ Tris- $\mathrm{Cl}(\mathrm{pH} 7.5)$, $150 \mathrm{mM} \mathrm{NaCl}, 2 \mathrm{mM}$ EDTA and 1\% SDS. The C-peptide concentration in the cells was determined by using a Human C-peptide ELISA Kit (Linco) and the total intracellular protein content was detected with a $\mathrm{BCA}^{\mathrm{TM}}$ Protein Assay Kit (PIERCE).

\section{Transplantation}

The Institutional Animal Care and Use Committees of Peking University approved all animal procedures. Prior to transplatation, Streptozotocin (STZ, Sigma) was injected i. p. at $40 \mathrm{mg} / \mathrm{kg} /$ day into 4-6 week-old BALB/c male nude mice for $5 \mathrm{~d}$ to induce experimental diabetes. Blood glucose was measured by GlucoTREND2 (Roche) from snipped tail. Only mice with blood glucose levels stably above $13.9 \mathrm{mM}$ after the STZ injections were used in these transplantation experiments. For transplantation, about $1 \times 10^{6}$ differentiated

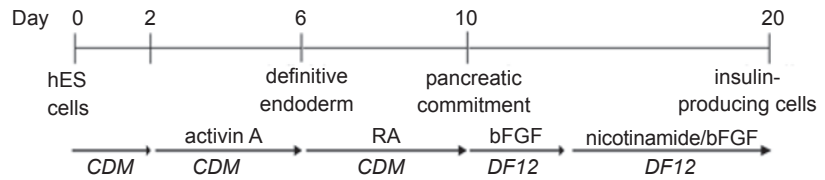

Figure 1 The sketch of human ES cell differentiation protocol. Undifferentiated human ES cells were first cultured in CDM for 2 $\mathrm{d}$ then transferred into CDM containing activin A for $4 \mathrm{~d}$. Then, the differentiated cells were further induced with RA in CDM for 4 dand transferred from CDM culture medium into DMEM/F12 islet maturation medium with bFGF added as a pancreatic cell maturation factor for $3 \mathrm{~d}$. Finally, the differentiated cells were switched to DMEM/F12 islet maturation medium containing bFGF and nicotinamide for another five days and transferred onto a hydrophobic plate in the same medium for a five-day suspension culture.
A 施
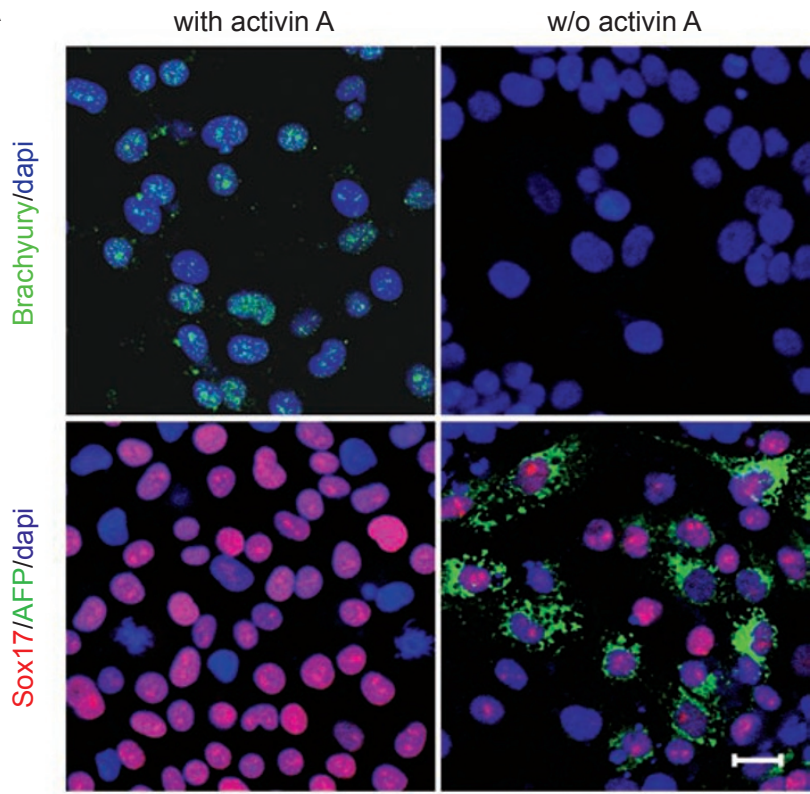

B

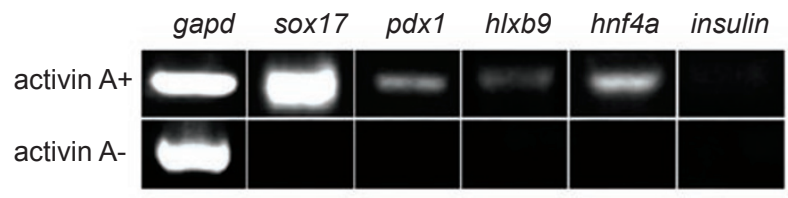

Figure 2 Activin A induced definitive endoderm differentiation from human ES cells. (A) After cultured in CDM with activin A for $4 \mathrm{~d}$, a number of cells expressing Brachyury and Sox 17 but not AFP appeared. However, without activin A induction, few Brachyurypositive cells and many Sox 17 and AFP double positive cells were detected (bar $20 \mu \mathrm{m}$ ). (B) Activin A treatment resulted in an increased expression of endoderm genes such as $\operatorname{sox} 17$ and $h n f 4 \alpha$.

cells in the final induction stage were injected into the left renal capsule. PBS or cells without activin A and RA induction were used as control. A glucose tolerance test was performed by i.p. injection of glucose (2.5 g/ kg body weight) after overnight fasting. After more than six weeks, the cell-transplanted kidneys were removed from those mice whose hyperglucemia was rescued after induced cell transplantation. Cryostat sections of the operated kidneys were prepared and C-peptide or pdx 1 expression of the transplanted cells in the renal capsule was confirmed by immunohistochemistry. The human ES-derived cells were detected by mouse anti-human nuclei monoclonal antibody (1:30, CHEMICON). Images were captured using an LEICA microscope DM4000B.

\section{Results}

Derive insulin-positive cells from human ES cells by the combination of activin $A, R A$ and maturation factors

Here we developed an effective method with activin A, 

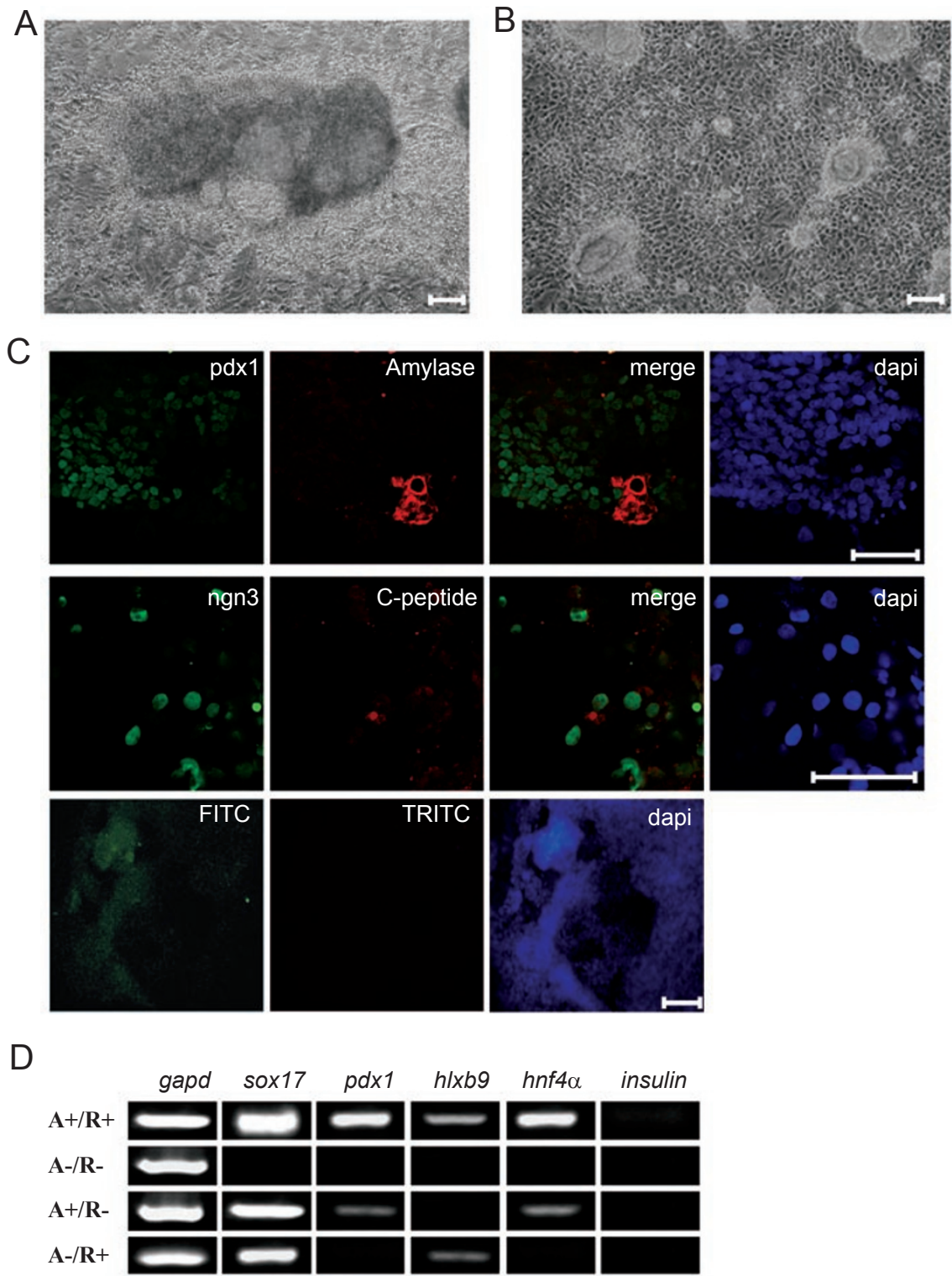

Figure 3 Pancreatic lineage differentiation from human ES cells was further induced with RA. (A) human ES cells cultured in CDM without activin A and RA treatment (bar $100 \mu \mathrm{m}$ ). (B) Small clusters formed from differentiated human ES cells in CDM with activin A and RA treatment (bar $100 \mu \mathrm{m})$. (C) pdx1 and Amylase staining, ngn3 and C-peptide staining of differentiated human ES cells in CDM after activin A and RA induction. Negative control staining of the differentiated human ES cells was shown in the lower panel (bar $40 \mu \mathrm{m}$ ). (D) With both activin A- and RA-induction, the mRNA levels of $p d x l, h n f 4 \alpha$ and $h l x b 9$ were induced as shown by RT-PCR detection. Without activin A and RA treatment, spontaneously differentiated human ES cells did not express $p d x 1, h n f 4 \alpha$ or $h l x b 9$. The presence of activin A alone induced only weak expression of $p d x l$ and $h n f 4 \alpha$ genes and RA alone induced only $h l x b 9$ expression.

RA and other maturation factors in serum-free medium to induce human ES cells to differentiate into functional insulin-producing cells. This induction process is illustrated in Figure 1. We first determined whether activin A could induce definitive endoderm formation from human ES cells. Undifferentiated $\mathrm{H} 1$ cells were passaged onto $1 \%$
Matrigel-coated dishes and cultured for $4 \mathrm{~d}$ in CDM system containing activin A. Results from our previous study [11], as well as the work of others [18-20], showed that high concentrations of activin A can lead to efficient endoderm differentiation. We tested with various concentrations of activin $\mathrm{A}$, and found that activin $\mathrm{A}$ at a concentration as 
A

B
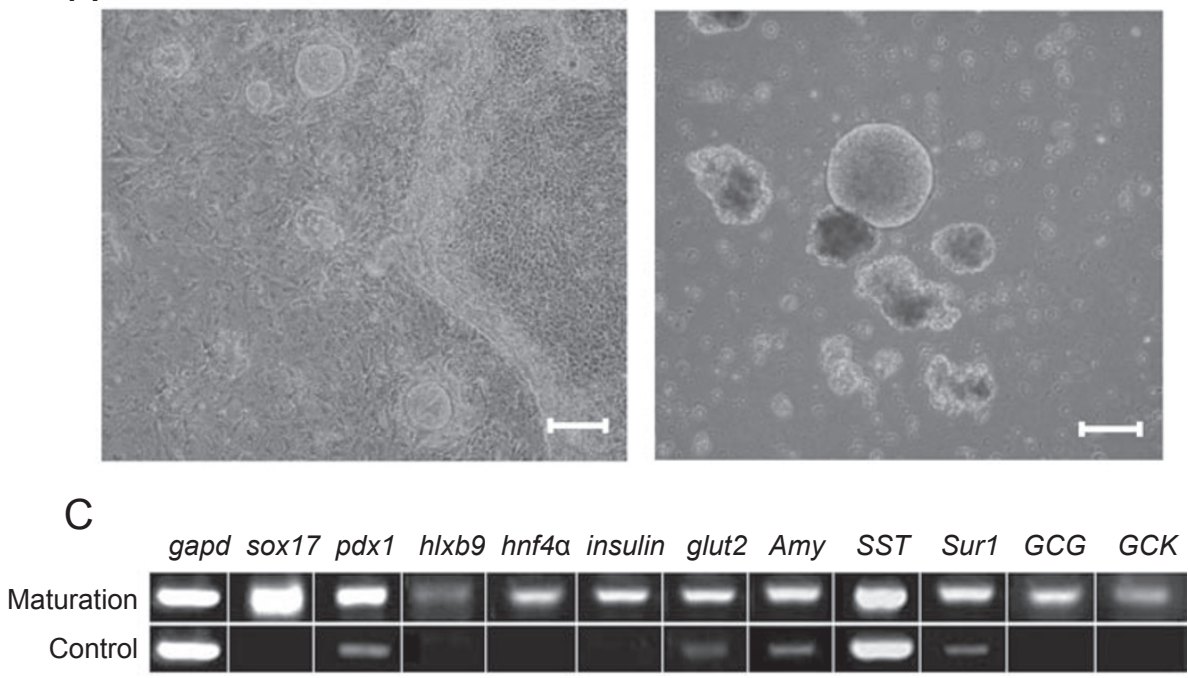

Figure 4 The cell morphology of the differentiated human ES cells at the final induction stage. The differentiated human ES cells expressed islet genes as detected by RT-PCR. (A) After activin A and RA induction, human ES cells were transferred into DMEM/ F12 islet maturation medium with bFGF and cultured in an adhesion plate for $3 \mathrm{~d}$. (B) Differentiated cells were transferred onto a hydrophobic plate for suspension culture and cultured for $3 \mathrm{~d}$ (bar $100 \mu \mathrm{m})$. (C) The differentiated human ES cells after the final induction step expressed islet genes, as detected by RT-PCR (upper panel); human ES cells differentiated without treatment by our induction method were used as control (lower panel).

low as $50 \mathrm{ng} / \mathrm{ml}$ was sufficient to induce human ES cells into endoderm lineages (data not shown). RT-PCR analyses revealed that activin A treatment at $50 \mathrm{ng} / \mathrm{ml}$ resulted in an increased expression of endoderm genes such as sox 17 and $h n f 4 \alpha$ (Figure 2B). Importantly, immunostaining further demonstrated that activin A caused a significant increase in the number of cells expressing Brachyury, a mesendoderm marker; and Sox17, an endoderm marker [14]. We also observed a decrease in the number of AFP-positive cells compared to a control experiment without activin A-induction (Figure 2A). Control staining of the differentiated human ES cells after activin A induction, omitting the primary antibody, was negative (data not shown).

Then, pancreatic lineage differentiation from human ES cells was further induced with $10^{-6} \mathrm{M}$ RA in CDM for another $4 \mathrm{~d}$. $10^{-6} \mathrm{M}$ RA was found to be sufficient for pancreatic progenitor differentiation from human ES cells, and higher concentration of RA could induce cell apoptosis (data not shown). At this stage, some cells formed clusters (Figure 3B). Immunofluorescence analysis revealed that in these differentiated cells, there were pdx 1 positive cells, which were not co-stained with the amylase-positive cells. In some cells, the expression of ngn 3 and the faint expression of C-peptide could be detected (Figure 3C). From three independent cultures, the percentage of $\mathrm{pdx} 1$ positive cells was on average $25 \%$. Control staining of the differ- entiated human ES cells after activin A and RA induction was performed as shown in Figure 3C. After both activin A and RA induction, the mRNA levels of $p d x 1, h n f 4 \alpha$ and $h l x b 9$, three transcription factors critical for early pancreas development, were shown by RT-PCR to have been induced (Figure 3D). In a control experiment without activin A and RA treatment, spontaneously differentiated human ES cells did not express $p d x 1, h n f 4 \alpha$ or $h l x b 9$. The presence of activin A alone induced only weak expression of the $p d x 1$ and $h n f 4 \alpha$ genes, while RA by itself induced only $h l x b 9$ expression. These results indicate that in the CDM culturing system, the sequential treatment of human ES cells with activin A and RA leads to more efficient differentiation into pancreatic cells.

Following the treatment with activin A and RA, we transferred the differentiated cells from CDM to the DMEM/F12 islet maturation medium containing bFGF [15] as a pancreatic cell maturation factor for three days (Figure 4A). Finally, the differentiated cells were switched to DMEM/F12 islet maturation medium containing nicotinamide [16] and bFGF for another three days and then transferred onto a hydrophobic plate in the same medium for a five-day suspension culture. In this final stage, many differentiated cells formed spherical clusters (Figure 4B) and expressed pancreatic $\beta$ cell markers such as $p d x 1$, insulin, glucokinase and glut2, as determined by RT-PCR 

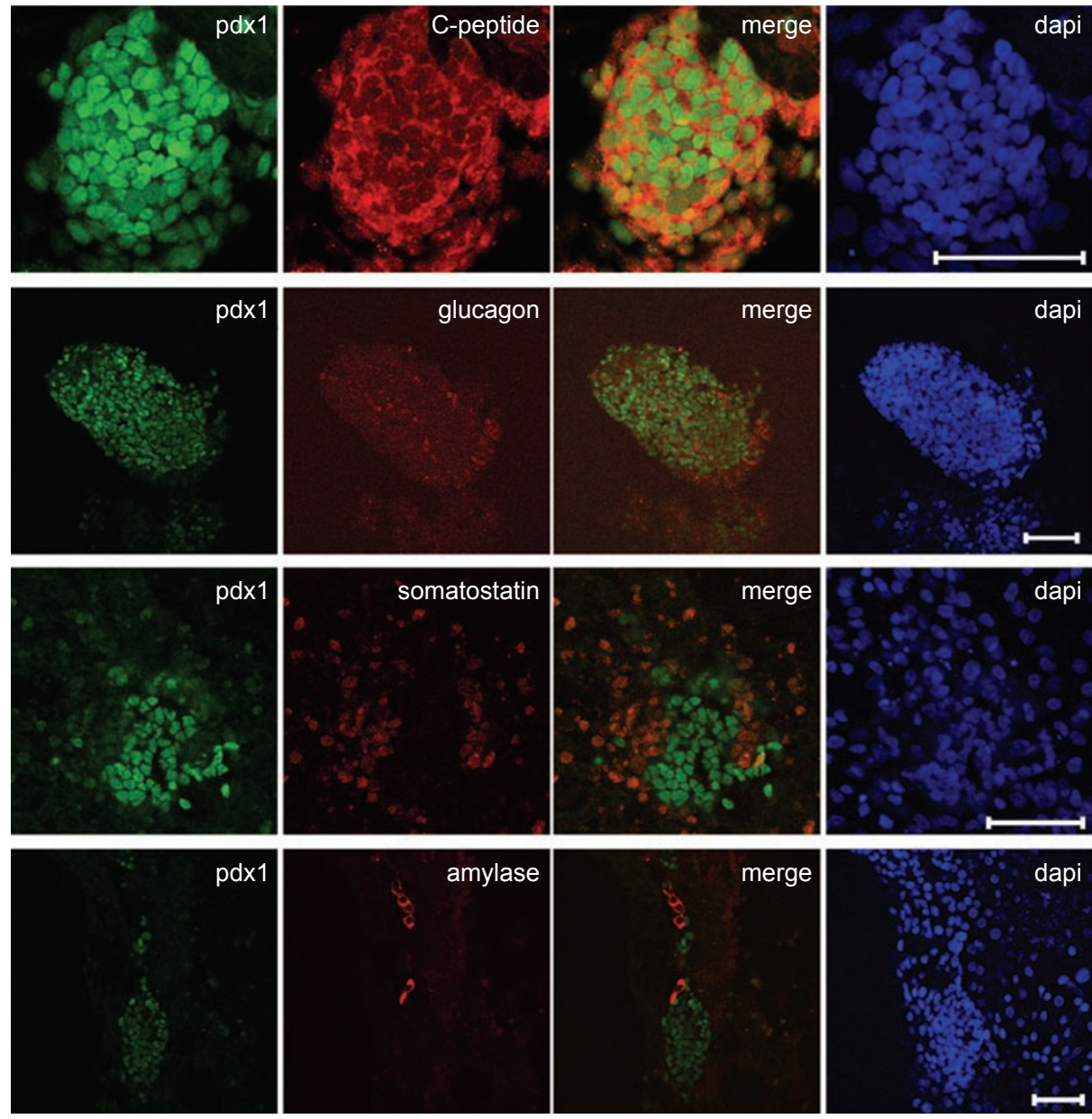

Figure 5 Immunostaining indicated that the terminally differentiated cells expressed pancreatic endocrine markers including Pdx1, C-peptide, Glucagon and Somatostatin and the exocrine marker Amylase (bar $50 \mu \mathrm{m}$ ).

analysis (Figure 4C). To detect whether these clusters expressed pancreatic specific markers such as C-peptide, glucagon and amylase, we plated the cells in suspension culture onto $1 \%$ Matrigel coated dishes to allow the aggregated clusters to adhere to a solid surface. These cells were then used for immunofluorescence analysis by confocal microscopy. As shown in Figure 5, among the differentiated cells, there were islet-like cluster structures and most of the cells in the clusters co-expressed C-peptide and pdx1, indicating that these cells were mature insulin-producing cells. In addition, immunostaining showed that within or around the $\mathrm{pdx} 1$ positive clusters, there were some $\mathrm{pdx} 1$ negative cells expressing pancreatic endocrine hormones such as glucagon or somatostatin. Some amylase positive cells were found near the pdx1 positive clusters, which suggested that pancreatic exocrine cells also existed in the differentiated cells after final induction. In the final stage, there were also $\mathrm{pdx} 1$ positive and C-peptide negative cells.
However, these cells were mostly in a monolayer and not in clustered structures, which could be still in the pancreatic progenitor stage (data not shown). In the islet-like clusters, there were a few $\mathrm{pdx} 1$ and somatostatin co-expressing cells; however, we did not find any expamples of cells that coexpressed $\mathrm{pdx} 1$ with either glucagon or amylase (Figure 5). To confirm whether there were any remaining completely undifferentiated cells, we checked the Oct4 expression by immunohistochemistry and found no Oct4-positive cells in the final induction stage (data not shown). Negative control staining of the differentiated human ES cells after the final stage of induction was also performed (data not shown).

As shown in Figure 6A, data collected from three independent cultures indicated that the percentage of $\mathrm{C}$ peptide-positive cells were more than $15 \%(16.9 \pm 4 \%)$ using our induction method. To exclude the possibility that apoptotic cells might take up insulin from the medium [10], we analyzed whether the insulin-producing cells were 
A

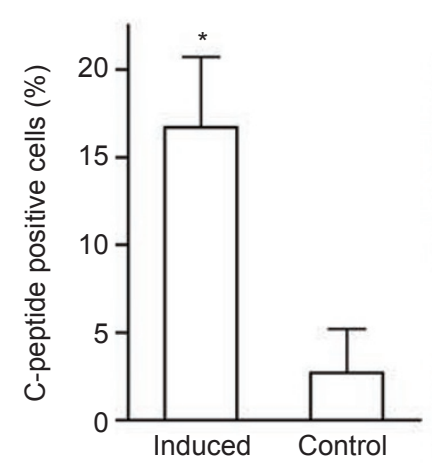

C
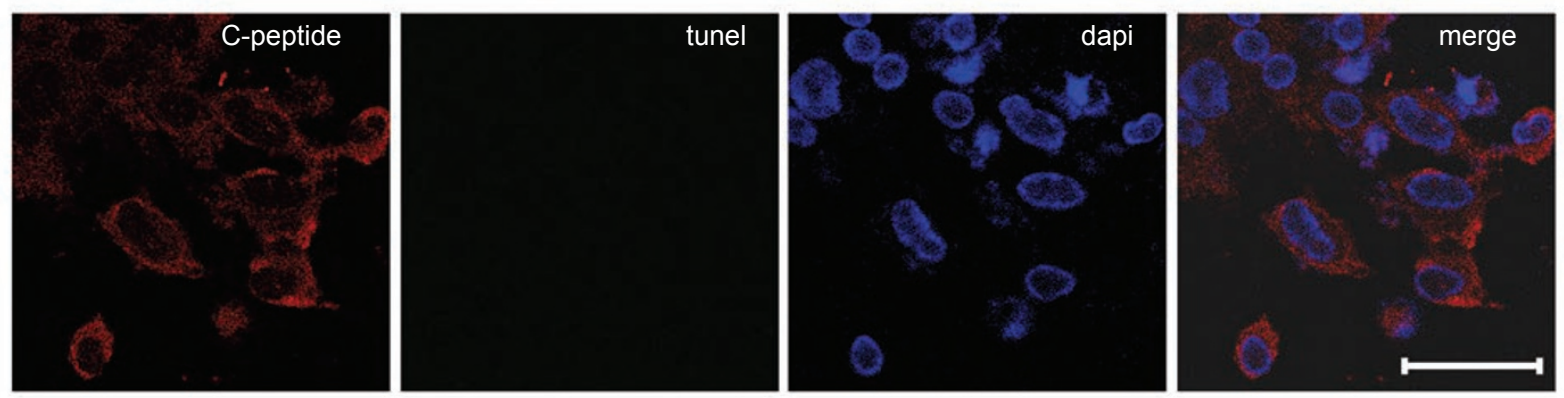
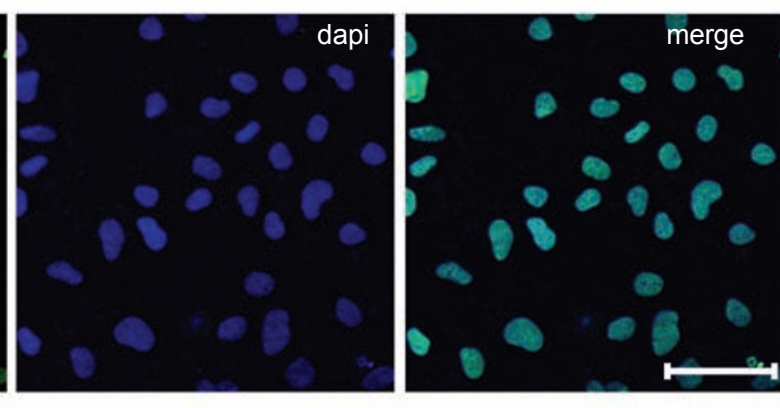

v
D

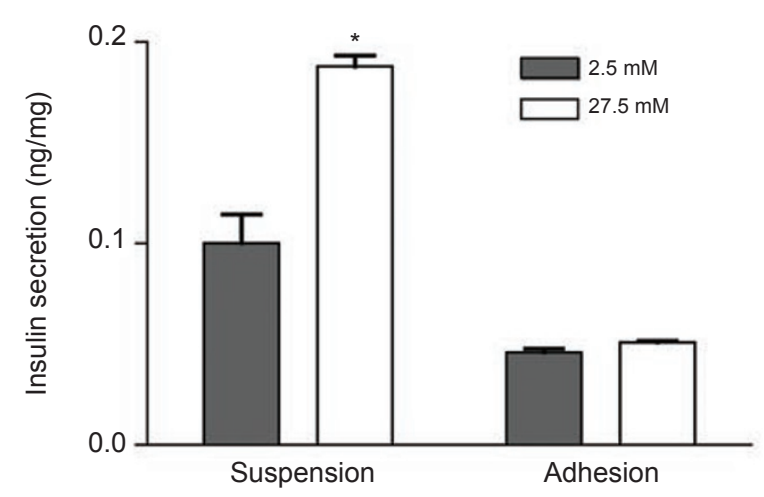

E

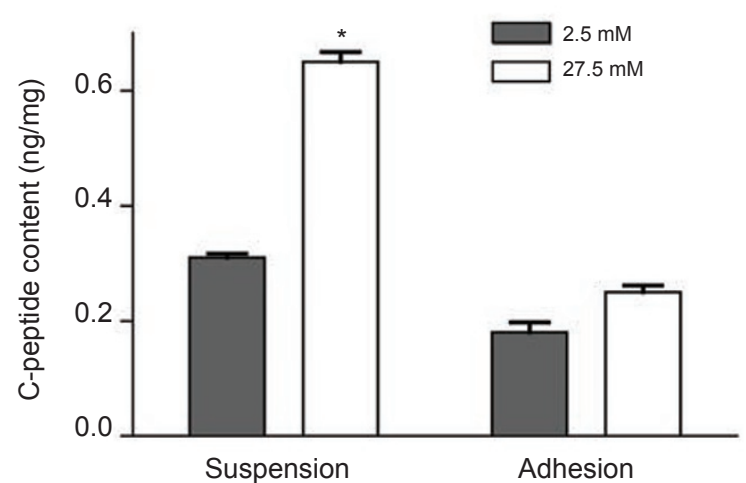

Figure 6 The C-peptide-positive cells in the final induction stage were TUNEL-negative and the percentage of C-peptide-positive cells exceeded $15 \%$. The secretion of insulin by the terminally differentiated cells was responsive to the glucose level. The intracellular C-peptide content of the differentiated cells was measured. (A) The statistics of C-peptide-positive cells within the differentiated cells in the final induction stage. (B) As a positive control, differentiated human ES cells were treated with DNase to induce apoptosis and stained with TUNEL. Data merged with DAPI (bar $40 \mu \mathrm{m})$. (C) There were no C-peptide-positive cells that co-stained with TUNEL, which indicated that the C-peptide-positive cells were not apoptotic (bar $40 \mu \mathrm{m}$ ). And the C-peptide showed clear cytoplasmic staining. (D) The secretion of insulin by the cells differentiated in suspension culture was responsive to the glucose level. However, insulin secretion from the cells in adhesion culture did not respond to glucose stimulation. (E) The intracellular C-peptide content of differentiated human ES cells was responsive to glucose content. *Data were analysed using a standard t-test where $P<0.05$ was considered to be significant. Insulin secretion (and C-peptide content) was compared between conditions where the cells were stimulated with either a low glucose concentration or a high glucose concentration. 
apoptotic using a TUNEL assay. We found no C-peptidepositive cells co-stained with TUNEL thus indicating that the C-peptide-positive cells were not apoptotic (Figure 6B and $6 \mathrm{C}$ ). Similar results were found in the differentiated cells derived from the H9 human ES cell line (data not shown).

\section{Insulin secretion by the induced cells responded to glucose stimulation}

To analyze whether the insulin secretion from these human ES cells derived insulin-producing cells was regulated by glucose, we treated the induced cells with Krebs-Ringer buffer containing low $(2.5 \mathrm{mM})$ or high $(27.5 \mathrm{mM})$ glucose concentrations, and then examined the insulin release in Krebs buffer by ELISA. We found that for the cells induced in the suspension culture, the insulin secretion was increased by $100 \%$ in the high glucose medium comparing to the low glucose medium (Figure 6D). We then lysed the differentiated cells and checked their intracellular C-peptide content. As shown in Figure 6E, the intracellular C-peptide content in suspension culture was also increased by $100 \%$ in the high glucose medium compared to the low glucose medium (Figure 6E). The intracellular C-peptide content in suspension culture was more than $0.35 \mathrm{ng} / \mathrm{mg}$, which further suggested that the insulin produced by these cells was not taken up from the culture medium. Comparing between suspension cultures and adhesion cultures (Figure $6 \mathrm{E})$, it is apparent that the suspension culture resulted in more efficient insulin production in the differentiated cells using our approach.

Transplantation of induced cell rescues hyperglycemia in thirty percent of the diabetic nude mice

To investigate whether the induced cells could function in vivo, we transplanted the differentiated cells under the renal capsules of diabetic nude mice. The blood glucose of $30 \%$ of the induced cell-transplanted mice $(n=6)$ was maintained at normal levels $(<13.9 \mathrm{mM})$ for nearly six weeks and the other $70 \%$ cell-transplanted mice $(n=13)$ maintained hyperglycemia. In the control groups, consisting of noninduced cell-transplanted $(\mathrm{n}=12)$ and PBS-transplanted $(n=11)$ mice, no mouse exhibited restoration of stable euglycemia. More than six weeks after transplantation, we removed the cell-transplanted kidney from the blood glucose rescued nude mice, and they regained hyperglycemia within three days after the operation and maintained it for at least two weeks (Figure 7A). The glucose tolerance test indicated that these $30 \%$ of nude mice with rescued blood glucose levels also attained improved glucose regulation capability compared to that of control-operated mice (Figure 7B).

To confirm that the human ES cell-derived beta cells could function in vivo, we first assayed for the presence of human ES cell-derived C-peptide or pdx 1 positive cells in the graft kidney. We observed that the C-peptide-positive cells and pdx1-positive cells were both co-stained by a human nuclei-specific antibody thus indicating that these C-peptide- or pdx1-positive cells were derived from the injected human ES cells (Figure 7C). We also detected the expression of glut 2 and $\mathrm{PC} 1 / 3$, two important functional markers, in the graft kidney by immunochemistry assay (Supplementary information, Figure S1). The C-peptide positive cells could also be detected in the renal capsules of the other $70 \%$ cell-transplanted mice that remained hyperglycemic, but at much fewer numbers than that in mice with rescued blood glucose levels (data not shown). When the differentiated hES cells without exposure to activin A and RA were transplanted, no C-peptide positive cells were detected after one month (Figure 7D). Moreover, we designed several human specific primers to further confirm the expression of human beta cell marker genes, including glucokinase, nkx6.1, IAPP, pax6 and Tcf1. Compared with the right kidney that did not receive cell transplantation, human specific $\beta$-actin and the beta cell marker genes were only expressed in the grafted left kidney, and their expressions were maintained even after more than two months post-transplantation (Figure 7E). These results showed that the human ES cell-derived insulin-producing cells were functional in vivo. Moreover, no Oct4-expressing cells were detected in the grafts (data not shown). And we followed these mice for three months post-transplantation and did not observe any teratoma formation.

We next assayed for blood C-peptide level in all of the recipients. Initially, we were unable to detect human C-peptide in mouse serum at both the fast and fed state. However, thirty minutes after glucose i.p. injection, we detected human C-peptide in serum but only in induced cell-transplanted mice $(0.5 \mathrm{ng} / \mathrm{ml}, \mathrm{n}=5)$. Although the level of human C-peptide detected in serum was quite low, these data showed that the induced cells generated by our protocol were functional in vivo.

\section{Discussion}

In this study, we have successfully developed a new approach for directing human ES cell differentiation into functional insulin producing cells within $20 \mathrm{~d}$. This newly developed method is based on our previous study, which had demonstrated that the combination of activin A and RA efficiently induces insulin-producing cells from mouse ES cells [11]. However, the previously established method for mouse ES cell differentiation is not directly applicable for human ES cells (data not shown). Several major improvements have been devised to establish an effective approach 
A

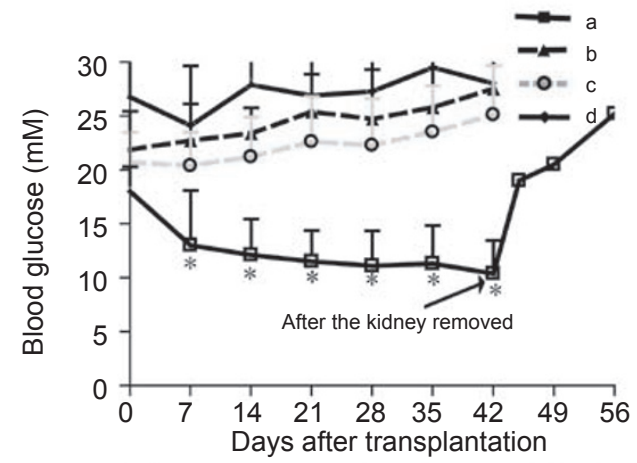

C
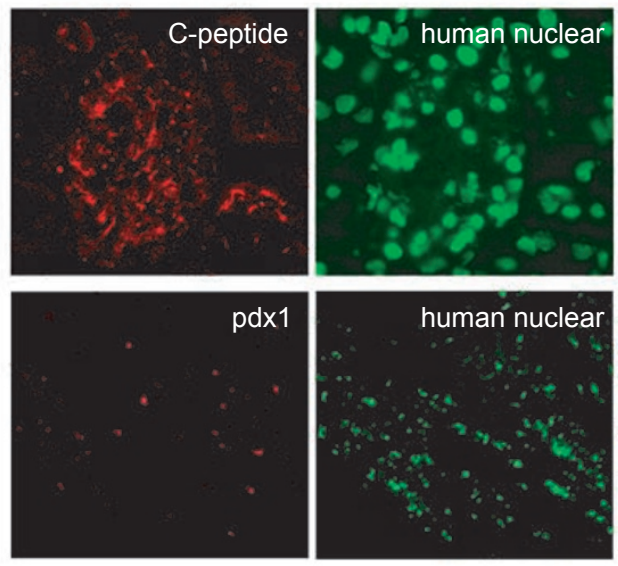

$\mathrm{D}$
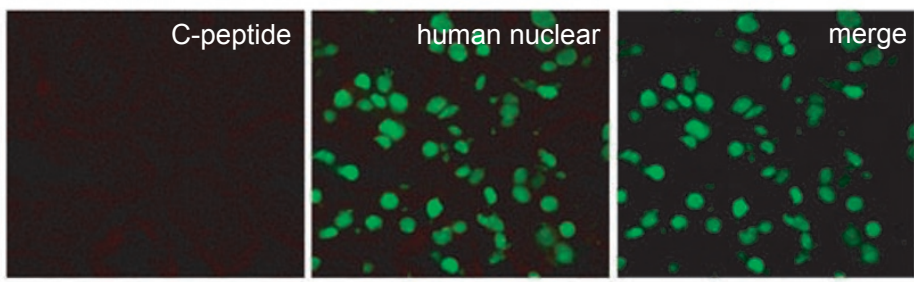

B
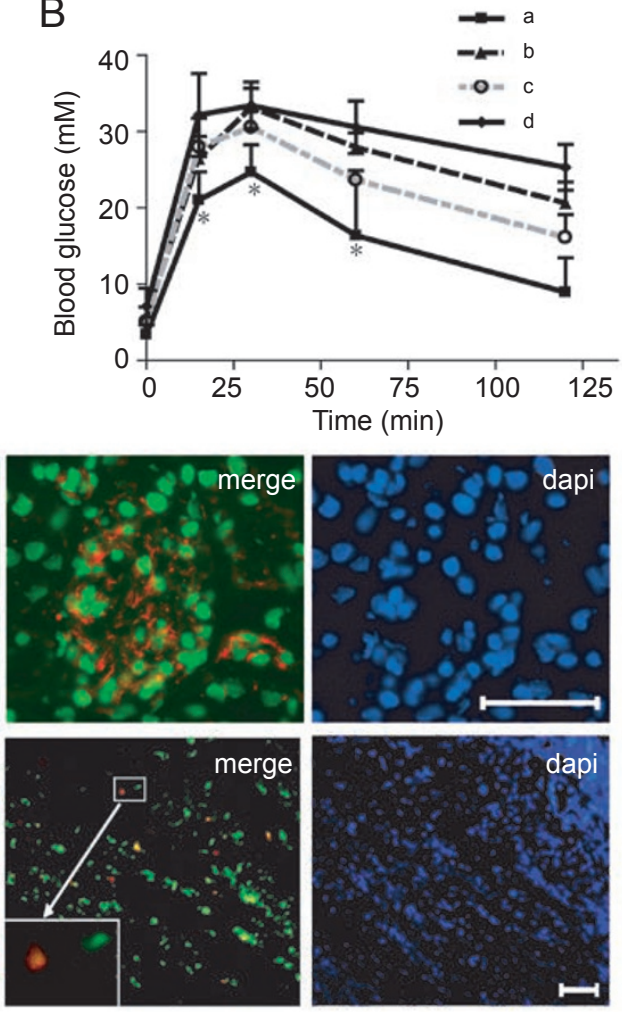

$E$

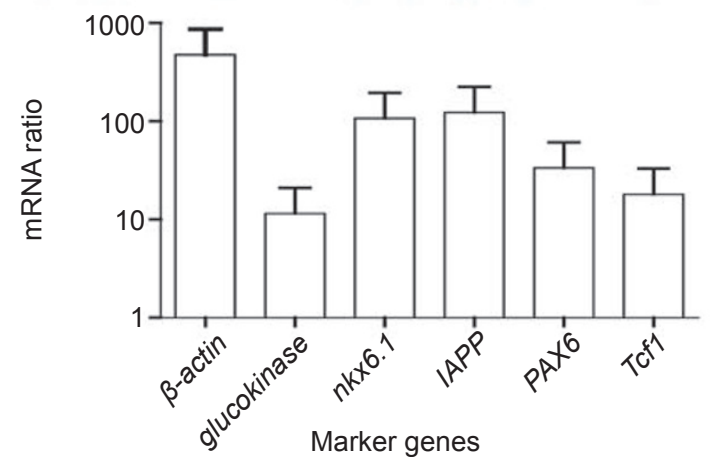

Figure 7 Transplantation of the induced human ES cells partially ameliorates diabetic symptoms. $\beta$ cell markers were detected in the kidney capsules transplanted with the cells induced by our approach. (A) The blood glucose test and (B) intraperitoneal glucose tolerance test of the operated mice transplanted with the induced cell, non-induced cell and PBS. (a, The $30 \%$ rescued diabetic nude mice, which were transplanted with induced human ES cells $(n=6)$. $b$, The other $70 \%$ induced cell-transplanted mice $(n=13)$ that remained hyperglycemic. c, The diabetic nude mice which were transplanted with the human ES cells without activinA and RA induction as control $(\mathrm{n}=12) . \mathrm{d}$, The diabetic nude mice which were only injected with PBS $(\mathrm{n}=11)) .{ }^{*}$ Data were analysed using a standard $\mathrm{t}$-test where $P<0.05$ was considered to be significant. The blood glucose (and glucose tolerance) of $30 \%$ of the induced cell-transplanted diabetic nude mice with the phenotypic rescue was compared to mice injected with PBS or transplanted with non-induced cells. (C) When the induced cells were transplanted into diabetic nude mice, C-peptide-positive and pdx1-positive cells derived from human ES cells were detected after six weeks. The C-peptide- or pdx1-positive cells were co-stained by a human nuclei-specific antibody (bar $=50 \mu \mathrm{m}$ ). (D) When the differentiated hES cells without activin A and RA induction were transplanted, almost no C-peptide positive cells were detected after one month (bar=50 $\mu \mathrm{m}$ ). (E) Human specific $\beta$-actin and beta cell markers were detected in the grafted left kidney. The results were normalized against the expression in the right kidney that did not receive cell transplantation. 
on human ES cells.

First, the entire induction process was performed in serum-free medium including the first two stages and a $\mathrm{DMEM} / \mathrm{F} 12$ islet maturation medium in the third stage. Initially, we failed to obtain definitive endoderm cells from human ES cells by using activin A in 10\% serum containing medium (data not shown). However, as we changed the induction process with the serum-free medium, the definitive endoderm marker Sox 17 [14] was detectable as shown in Figure 2B, which indicated that activin A was effective in inducing definitive endoderm differentiation from human ES cells. The serum-free culturing medium avoided the negative effects of unknown factors in the serum on human ES cell differentiation, and also made it possible to more effectively study the precise effects of different growth factors or signaling pathways on human ES cell differentiation.

Second, using our approach, we found that a suspension culture was apparently better for beta cell maturation than an adherent culture based on our insulin secretion and intracellular C-peptide content test. Interestingly, a similar effect has been observed in human islet cell culture in $v i$ tro. Lechner A et al reported that culturing of human islet cells in a monolayer leads to the loss of insulin expression and that re-aggregation of the expanded islet cells was subsequently required to increase insulin expression in vitro [17].

Our previous study [11] and the results presented here show that the combination of activin A and RA can effectively induce both mouse and human ES cells to differentiate into insulin-producing cells. The individual effect of activin A and RA on endoderm and pancreas development has also been demonstrated by several other groups [18-24]. It has been shown that mouse and human ES cells can be efficiently induced into Sox 17-positive definitive endoderm by activin A treatment [18-20]. RA has been reported to be critical for early pancreas formation during zebrafish and mouse embryonic development [21-24]. In our study, as shown in Figure 3, when induced by activin A alone, human ES cells weakly express the pancreatic early transcription factors $p d x l$ and $h n f 4 a$. With RA induction alone, human ES cells express only $h l x b 9$. However, when human ES cells were induced with a combination of activin $\mathrm{A}$ and RA in sequence, the expression of $h l x b 9, p d x l$ and $h n f 4 a$ were all increased significantly. These results suggest that the combination of activin A and RA is an effective method to promote pancreas specification from human ES cells, which mimics the in vivo pancreatic development pathway, i.e. from blastocyst to endoderm and then from endoderm to pancreas specification.

When human ES cells induced by our stepwise approach were transplanted into STZ-treated diabetic nude mice, beta cell markers including C-peptide, pdx1, glut2, PC1/3, glucokinase, nkx6.1, IAPP, pax6 and Tcf1 were detected in kidney capsules six weeks later, indicating that the insulin-producing cells could successfully survive in vivo. We further showed that via injection into diabetic nude mice the induced human ES cells could rescue the hyperglycemia phenotype. The blood glucose levels of all the operated mice showed an obvious decrease in the first week compared to the control, and $30 \%$ of the operated mice subsequently exhibited stable euglycemia for more than six weeks. This $30 \%$ rescue rate might be due to two reasons. First, the procedure involved xenotransplantation such that human cells were injected into the renal capsules of nude mice. The physiological differences between human and mice could very likely impair the function of transplanted human ES cell-derived islet-like cells. Second, even for the model involving the transplantation of human islet into nude mice, islet purity and insulin content are both critical for proper graft function [28]. The presence of exocrine tissue in islet preparations has been previously shown to impair islet engraftment in nude mice [26]. Impure islets have been shown to induce tissue necrosis and subsequent fibrosis at the implant site, consequently delaying the revascularization process [27]. In the approach described here, there are still pancreatic exocrine cells within the induced islet-like clusters that might affect the function of the transplanted cells. Therefore, further work is needed to purify the pancreatic beta cells from the induced human ES cells so as to improve the differentiation efficiency for successful transplantation.

Our present protocol was designed to mimic pancreatic islet development and included three developmental events: definitive endoderm formation, pancreas specification and endocrinal islet maturation. Our data suggests that strategies which aim to mimic in vivo organogenesis should be a promising approach for differentiating ES cells into pancreatic islet cells. D' Amour et al. also reported a complicated stepwise protocol [10], from which they obtained pancreatic endocrine hormone-expressing cells. In their studies, insulin-expressing cells were selected by using the zinc-chelating dye dithizone. These purified dithizone-stained cells had an insulin content approaching that of adult islets. However, C-peptide release from the cells induced by their protocol did not respond well to glucose stimulation. Furthermore, many immature cells that co-expressed insulin and glucagon were also present. Moreover, in vivo functional transplantation studies were not performed. In our present work, the secretion of insulin and the production of C-peptide from the cells induced by our protocol corresponded to the variations in glucose levels used in our studies. Also, no pdx1 and glucagon co-expressing cells were observed. Transplanta- 
tion of these induced cells into diabetic mice was able to partially rescue the recipients, and the expression of several beta cell marker genes could be observed in the graft even after more than two months post-transplantation. The rate of C-peptide-positive cells was approximately $15 \%$ and the rescue efficiency was approximately $30 \%$, suggesting that further improvement is needed.

In summary, an effective stepwise approach utilizing the combination of activin A, RA and other maturation factors has been shown to induce human ES cells to differentiate into functional insulin-producing cells, and these differentiated cells are able to rescue diabetic nude mice to a certain extent after transplantation. This approach offers a promising in vitro model for studying human pancreas development and also helps fulfill the urgent need of an ample supply of insulin-producing cells for cell transplantation therapy in type I diabetes.

\section{Acknowledgments}

This research was supported by the Ministry of Science and Technology Grant (2001CB510106), Science and Technology Plan of Beijing Municipal Government (H020220050290), National Natural Science Foundation of China Awards for Outstanding Young Scientists (30125022) and for Creative Research Groups (30421004), and Bill \& Melinda Gates Foundation Grant (37871) to H Deng. We thank Dr C Wright for kindly providing us with the pdx 1 antibody. We thank Drs Matt Stremlau, Tung-Tien Sun, Johnny He, and Hui Zhang for critical reading of the manuscript. We thank Yizhe Zhang for technical support on real-time PCR. We also thank Donghui Zhang, Xulei Sun, Yushan Guo, Zan Tong, Jun Cai, Han Qin, Chengyan Wang, Pengbo Zhang, Chunbo Teng, Jiefang You, Yan Shen, Wei Wei and other colleagues in our laboratory for technical assistance and advice during experiments.

\section{References}

1 Shapiro AM, Lakey JR, Ryan EA, et al. Islet transplantation in seven patients with type 1 diabetes mellitus using a glucocorticoid-free immunosuppressive regimen. N Engl J Med 2000; 343:230-238.

2 Hussain MA, Theise ND. Stem-cell therapy for diabetes mellitus. Lancet 2004; 364:203-205.

3 Thomson JA, Itskovitz-Eldor J, Shapiro SS, et al. Embryonic stem cell lines derived from human blastocysts. Science 1998; 282:1145-1147.

4 Cowan CA, Klimanskaya I, McMahon J, et al. Derivation of embryonic stem-cell lines from human blastocysts. N Engl J Med 2004; 350:1353-1356.

5 Assady S, Maor G, Amit M, et al. Insulin production by human embryonic stem cells. Diabetes 2001; 50:1691-1697.

6 Brolén G, Heins N, Edsbagge J, et al. Signals from the embryonic mouse pancreas induce differentiation of human embryonic stem cells into insulin-producing beta-cell-like cells. Diabetes 2005; 54:2867-2874.

7 Lumelsky N, Blondel O, Laeng P, et al. Differentiation of embryonic stem cells to insulin-secreting structures similar to pancreatic islets. Science 2001; 292:1389-1394.

8 Segev H, Fishman B, Ziskind A, et al. Differentiation of human embryonic stem cells into insulin-producing clusters. Stem Cells 2004; 22:265-274.

9 Hansson M, Tonning A, Frandsen U, et al. Artifactual insulin release from differentiated embryonic stem cells. Diabetes 2004; 53:2603-2609.

10 D'Amour KA, Bang AG, Eliazer S, et al. Production of pancreatic hormone-expressing endocrine cells from human embryonic stem cells. Nature Biotechnol 2006; 24:1392-1401.

11 Shi Y, Hou L, Tang F, et al. Inducing embryonic stem cells to differentiate into pancreatic â cells by a novel three-step approach with activin A and all-trans retinoic acid. Stem Cells 2005; 23:656-662.

12 Johansson, BM, Wiles MV. Evidence for involvement of Activin A and bone morphogenetic protein 4 in mammalian mesoderm and hematopoietic development. Mol Cell Biol 1995; 15:141151.

13 Gao R, Ustinov J, Pulkkinen MA, et al. Characterization of endocrine progenitor cells and critical factors for their differentiation in human adult pancreatic cell culture. Diabetes 2003; 52:2007-2015.

14 Kanai-Azuma M, Kanai Y, Gad JM, et al. Depletion of definitive gut endoderm in Sox17-null mutant mice. Development 2002; 129:2367-2379.

15 Hardikar AA, Marcus-Samuels B, Geras-Raaka E, et al. Human pancreatic precursor cells secrete FGF2 to stimulate clustering into hormone-expressing islet-like cell aggregates. Proc Natl Acad Sci USA 2003; 100:7117-7122.

16 Otonkoski T, Beattie GM, Mally MI, et al. Nicotinamide is a potent inducer of endocrine differentiation in cultured human fetal pancreatic cells. J Clin Invest 1993; 92:1459-1466.

17 Lechner A, Nolan AL, Blacken RA, et al. Redifferentiation of insulin-secreting cells after in vitro expansion of adult human pancreatic islet tissue. BBRC 2005; 327:581-588.

18 Kubo A, Shinozaki K, Shannon JM, et al. Development of definitive endoderm from embryonic stem cells in culture. Development 2004; 131:1651-1662.

19 Tada S, Era T, Furusawa C, et al. Characterization of mesendoderm: a diverging point of the definitive endoderm and mesoderm in embryonic stem cell differentiation culture. Development 2005; 132:4363-4374.

20 D'Amour KA, Agulnick AD, Eliazer S, et al. Efficient differentiation of human embryonic stem cells to definitive endoderm. Nature Biotechnol 2005; 23:1534-41.

21 Stafford D, Prince VE. Retinoic Acid signaling is required for a critical early step in Zebrafish pancreatic development. Current Biology 2002; 12:1215-1220.

22 Stafford D, White RJ, Kinkel MD. Retinoids signal directly to zebrafish endoderm to specify insulin-expressing cells. Development 2006; 133:949-956.

23 Martin M, Gallego-Llamas J, Ribes V, et al. Dorsal pancreas agenesis in retinoic acid-deficient Raldh2 mutant mice. Dev Biol 2005; 284:399-411. 
24 Micallef SJ, Janes ME, Knezevic K, et al. Retinoic acid induces pdx1-positive endoderm in differentiating mouse embryonic stem cells. Diabetes 2005;54:301-305.

25 Migliavacca B, Nano R, Antonioli B, et al. Identification of in vitro parameters predictive of graft function: A study in an animal model of islet transplantation. Transplant Proc 2004; 36:612-613.
26 Gray DW, Sutton R, McShane P, et al. Exocrine contamination impairs implantation of pancreatic islets transplanted beneath the kidney capsule. J Surg Res 1988; 45:432-442.

27 Mattsson G, Jansson L, Nordin A, et al. Impaired revascularization of transplanted mouse pancreatic islets is chronic and glucose-independent. Transplantation 2003; 75:736-739. 